\title{
That mighty pantun river and its tributaries
}

\author{
DING CHOO MING
}

\begin{abstract}
Known as pantun to the Malays in Brunei, Malaysia, Pattani, Riau, Singapore, and Southern Phillipines, it is called peparikan to the Javanese, sesindiran to the Sundanese and many other different names in different ethnic groups in the different parts of the Indo-Malay world, which is made up of Brunei, Indonesia, Malaysia, Singapore, Pattani in southern Thailand, and Mindanao in the southern Philippines. In almost every settlement that sprang up along the major rivers and tributaries in the Indo-Malay world, the pantun blend well with their natural and cultural surroundings. In this article, the geographical extent of the pantun family in the Indo-Malay world is likened to a mighty river that has a complex network of tributaries all over the Indo-Malay world. Within the Indo-Malay world, it is the movement of the peoples help the spread of pantun from one area to the other and makes it an art form of immensely rich and intricate as can be seen from the examples given.
\end{abstract}

KEYWORDS

Pantun, oral poetry, Indo-Malay identity, pantun distribution, Anglo-Dutch Treaty of 1824 .

\section{ONE BIG FAMILY NETWORK}

Pantun emerged somewhere in the Indo-Malay world long before the arrival of Hinduism some 1,500 years before Islam came to the area in the thirteenth century. As a form of oral poetry, pantun were originally composed and transmitted without being written down. Following the disappearance of oral culture, more and more pantun are now composed and transmitted not only in written, but also in printed and even in digital forms. This leads to a situation where written versions of pantun not only influence their memorized versions,

DING CHOO MING is Principal Research Fellow at the Institute of the Malay World and Civilization, The National University of Malaysia, 43600 UKM, Bangi. His theoretical interests lie in authorship of Malay literature in Riau-Lingga empire, the authorship and creavitity of pantun in the Malay-Indonesia world, and the development of indigenous information in the Malay world (http:/ / malaycivilization.ukm.my). Among his recent books are Manuskrip Melayu Sumber Maklumat Peribumi Melayu (2008) and Pengajian di Alam Melayu: Dari Tradisi Manuskrip ke Maklumat Digital (2009). Ding Choo Ming may be contacted at: dingukm@yahoo.com.

(C) 2010 Faculty of Humanities, University of Indonesia 
but also that the survival of pantun increasingly depends on writing and printing, much like all other memorized oral poetry, including nursery rhymes, peribahasa, and kata adat that all have come under the spell of writing, printing, and digital technologies. The complex relationships between oral, writing, printing, and digital technologies make pantun not only geographically the most widely dispersed, but also historically the best established folk poetry (Winstedt 1969). In almost every settlement that sprang up along the major rivers and tributaries in Brunei, Indonesia, Malaysia, Singapore, Pattani in southern Thailand, and Mindanao in the southern Philippines, pantun blend well with their natural and cultural surroundings. In this article, the geographical extent of the pantun family in Indonesia, Malaysia, Singapore, Brunei, the southern Philippines and Thailand is likened to a mighty river that has a complex network of tributaries all over the Indo-Malay world. Though we have no trace of its source in historical and geographical terms, our fascination with its source remains as intense as it has been since the dawn of Indo-Malay civilization and history, when the ancestors of the Indo-Malays scratched a living from the rich and fertile soil along the banks of major rivers and tributaries.

The continual outpour of new pantun has caused the mighty pantun river to build up. Millions of people living along the banks of all the major rivers have enjoyed and celebrated their own pantun at various occasions. In this way, pantun have been elevated in position, importance, and popularity, towering high above all other forms of poetry, be they syair, gurindam, or seloka, which are not only non-indigenous but also appear much later in history. For centuries, the Indo-Malay peoples have adored and embraced their pantun because of their intrinsic value and their antiquity, importance, popularity, and influence upon them (Daillie 1968). This is evident from the way pantun have been used with good reason to grace auspicious and grand occasions and ceremonies and more importantly as a tool in education, entertainment, information, and wisdom. One way or another, directly or indirectly, the pantun river has nourished Indo-Malay language, civilization, culture, and history. Over the years, we have collected some 120,000 different pantun in different languages (and dialects) from different regions in the Indo-Malay world. Although they are in different languages, dialects and called by different names, most of these co-called pantun share the same structure, characteristics, and functions, and hence provide a strong bond between the different ethnic and dialectal groups living in the Indo-Malay world. This development has recently prompted me to undertake research under an Asian Public Intellectual grant on the role of the pantun as yet another Indo-Malay regional cultural identity (Ding Choo Ming 2008b). It is the spatial distribution of the pantun that forms the focus of this paper.

\section{THE ANALOGY OF PANTUN RIVER}

Muhammad Haji Salleh (2006) likens the complex pantun family to a tree with different flowers. To me, the spatial distribution of the pantun is more 
appropriately compared with a mighty cultural river with its tributaries and given different names all over Indo-Malay world (Ding Choo Ming 2008c). As one of the pillars of Indo-Malay civilization, pantun are certainly sources of the wisdom of the Indo-Malay peoples. Rising deep in the history of the past, it loops and twists across the Indo-Malay world comprising 30,000 islands with 350 million people in Indonesia, Malaysia, Singapore, Brunei, the southern Philippines, and Thailand. This vast area was one entity before the Dutch and British colonial masters divided it into their colonies following the Anglo-Dutch Treaty in 1824. As a mighty cultural river, pantun traverse all the villages, towns, ports, and other settlements be they in lowland, coastal areas, valleys, hills, or mountains on its 50,000 kilometre journey from the east to the west and some 10,000 kilometres from the north to the south. From the air, we see that different indigenous ethnic groups speaking different language and dialects along the different stretches of the cultural river have co-existed with their versions of pantun. Throughout their long existence, the richness of the pantun has provided them with wisdom, guidance, values, education, and entertainment to sustain their cultural identity and their sense of belonging. The swirling flow of the pantun river has brought survival and pride to these peoples until today. This pantun river, which represents the primordial IndoMalay culture, will certainly reach out into the infinite future because of their power, magic, splendour, pride, and many other wonders.

Physically, the Indo-Malay world is pre-eminently water-dominated where rivers and seas not only constitute major highways, but also form the major economic life-lines for the peoples, giving rise to the self subsistence economy of the peasantry that merges into the market-centred economy and in trading in the ports and towns near the coastlines (Tweddell and Kimball 1985: 27). In addition, the complex river network and trade lead to the dissemination of the pantun all over the Indo-Malay world. Over the centuries, many people from Palembang, Ambon, Pontianak, and Malacca, for instance, moved to Kedah, Perak, Pahang and many other faraway places, to and fro, for various reasons, including marriage, migration, work, and education. This allowed the pantun of Palembang, Ambon, Pontianak, Malacca and many others to be brought to Pahang, Kedah, and Perak so that they could be enjoyed not only in their homelands, but also elsewhere. This means that the pantun and the movement of the people are intricately linked through history. This means too that though no longer living in their ancestral homelands, the descendents of these IndoMalay peoples shared their pantun with friends and neighbours who were not necessarily family members or member of their communities in their adopted lands. Now, peoples living in different localities, away from their homelands, share feelings of togetherness because of the culture they brought with them. This feeling of togetherness transcends geographical boundaries.

\section{INTERDEPENDENT OR INDEPENDENT DEVELOPMENT?}

The word pantun is commonly used in most of the Malay languages and dialects in Malaysia, Brunei, Singapore, Pattani, and many regions in Indonesia. 
Thus, the word pantun is used in the Alas and the Banjarese dialects, while in Acehnese, the word pantun refers to the same form of poem. In Kerinci, it is panton, and in Manado, it is pantung. However, in Toba-Batak, they are called ende-ende or umpasa. Muhammad Haji Salleh and Bazrul Bahaman (1999; http://www.usm.my/pantun/pantuninenglish1.asp) have collected pantun from 39 dialects of Malay and 25 non-Malay languages. Their distribution can be seen in Chart I.

\begin{tabular}{|c|c|c|c|}
\hline & & Pantun in Malay languages & $\begin{array}{l}\text { Pantun in Non- } \\
\text { Malay language }\end{array}$ \\
\hline 1 & Region & Areas & Areas \\
\hline 2 & Sumatra & $\begin{array}{l}\text { Aceh, Langkat/ Deli/ Serdang, } \\
\text { Jambi, Minangkabau, Kerinci, } \\
\text { Insular Riau (Bengkalis, } \\
\text { Penyengat), Rokan, Indragiri } \\
\text { Hulu, Kampar, Proto-Malays, } \\
\text { Ogan Komering, Lahad, Basemah, } \\
\text { Musi, Prabumulih, Muara Enim, } \\
\text { Lampung, Serawai. }\end{array}$ & $\begin{array}{l}\text { Karo, Toba, } \\
\text { Mandailing, } \\
\text { Simalungun, } \\
\text { Acehnese, Gayo, } \\
\text { Alas. }\end{array}$ \\
\hline 3 & Kalimantan & $\begin{array}{l}\text { Sintang, Ketapang, Sambas, Hulu } \\
\text { Kapuas, Kutai, Banjar, Pasir. }\end{array}$ & \\
\hline 4 & Sulawesi & Manado, Gorontalo. & $\begin{array}{l}\text { Gorontalo, } \\
\text { Sangihe-Talaud, } \\
\text { Minahasa. }\end{array}$ \\
\hline 5 & Maluku & Ambon, Ternate. & - \\
\hline 6 & Java & Jakarta, peranakan Tionghoa. & \begin{tabular}{|l|} 
Javanese, \\
Sundanese.
\end{tabular} \\
\hline 7 & Malaysia & $\begin{array}{l}\text { Kedah, Perlis, Pulau Pinang, } \\
\text { Kelantan, Terengganu, Johor, } \\
\text { Pahang, Melaka, Selangor, Perak, } \\
\text { Negeri Sembilan, Sabah, Sarawak, } \\
\text { Peranakan Cina, Peranakan India } \\
\text { (Ceti), Semak Brik. }\end{array}$ & $\begin{array}{l}\text { Iban, Bidayuh, } \\
\text { Melanau, Kedayan, } \\
\text { Bajau. }\end{array}$ \\
\hline 8 & Southern Thailand & Pattani. & \\
\hline 9 & Bali & & Bali Aga. \\
\hline 10 & West Nusa Tenggara & & Sasak. \\
\hline
\end{tabular}

Chart I: The distribution of pantun in the Indo-Malay world.

From my documentation on pantun in Aceh, Ambon, Banjarmasin, Belitung, Bugis, Dayak, Jakarta (particularly Melayu-Betawi), Kalimantan Barat, Kerinci, Lampung Pubiyan, Langkat, Maluku, Malacca (particularly Baba and Chetti) (Ding Choo Ming 2008a, 2008b), Makassar (particularly Melayu Makassar), Mandar, Minangkabau, Muna, Pontianak, Saluan, Sangau, Seluma, Serdang, Talang Mamak, Tanemperar, Ternate, Toraja, Totoli, and Tetun, I come across eighteen different names given to pantun. They include kalang in Bajau, wewangsalan in Bali, umpasa or ende-ende among the Simalungun 
Batak, pamiula amongst the Iranun, parikan and sometimes kentrung in Java, sudawil in Kadazandusun, badendang kapata in Maluku, pantung in Manado, kalindaqdaq in Mandar, panuntun (sometimes) in Minangkabau, kabanti in Muna, nyuriah in Seluma, cuit-cuitan or rendai in Serawai, sisindiran in Sunda and londe in Toraja. This list is certainly far from complete as there are many other pantun from many other areas that yet have to be collected. There are approximately 400 major and minor languages in the Indo-Malay world, ranging from Acehnese, Batak and Minangkabau in the westernmost part of Indonesia to the languages of Papua, 3,500 miles away in the East (Sastrowardoyo and Damono 1985: ix- x). One immediate impression is that the diversity of the pantun in the Indo-Malay world reflects the diversity of languages and cultures that exist. Looking around, one immediate question is, are there independent developments or minor traditions in the big pantun family? As we have no clue on their history and development, we also have no idea which "regional pantun" should be considered as the earliest to emerge and to flourish. As mentioned earlier, in almost every settlement, pantun are inextricably linked with the peoples and their surroundings. Over time these ethnic and dialectical pantun have all risen to the status of regional and ethnic pride. However, over the years, if not centuries, the massive movement of the Indo-Malay peoples speaking different dialects and languages has resulted in Palembang, Minangkabau, Acehnese, Makassarese, Pontianak, and Malaccan pantun to flourish outside their homelands. Their meeting and co-existence in their new and adopted lands and islands changed the flow of these pantun geographically. The answer to the question whether there are independent developments or minor traditions in the big pantun family awaits further exploration. In the meantime, it is not wrong to compare the various pantun from different regions among the tributaries of the mighty pantun river of Indo-Malay world.

The basic structure of the pantun is the same virtually everywhere. Rhymed $\mathrm{a} b \mathrm{a} b$ in a four-line pantun, the first two lines form the so-called foreshadow (usually depicting nature), and compliments the next two lines, called the meaning (usually metaphoric in nature). See some examples in Chart II.

\begin{tabular}{|l|l|l|l|}
\hline No & Region & Example 1 & Example 2 \\
\hline 1 & Aceh & $\begin{array}{l}\text { Tajak ublang tajak pot campli } \\
\text { Pasoe lam guni ba u langsa } \\
\text { Nyum sang lon jeuh ie mon } \\
\text { hayati } \\
\text { Laupie that atee ngon lon ngieng } \\
\text { gata }\end{array}$ & $\begin{array}{l}\text { Koh on pisang tabalot } \\
\text { payeh } \\
\text { Ranub seulaseh di dalam } \\
\text { baling } \\
\text { Bak malam nyoe reungent } \\
\text { that lopn eeh } \\
\text { Ke meuwoe gaseh loon } \\
\text { ngon cutbang }\end{array}$ \\
\hline 2 & Ambon & $\begin{array}{l}\text { Kayu titi kayu gelaga } \\
\text { Tidak sama kayu di Ambon } \\
\text { Mama tiri mama piara } \\
\text { Tidak sama yang gandong }\end{array}$ & $\begin{array}{l}\text { Pukul sagu di tana rata } \\
\text { Buang ela di alor miring } \\
\text { Rindu itu deng ingat bapa } \\
\text { Air mata jato di piring }\end{array}$ \\
\hline
\end{tabular}




\begin{tabular}{|c|c|c|c|}
\hline No & Region & Example 1 & Example 2 \\
\hline 3 & $\begin{array}{l}\text { Baba } \\
\text { (Melaka) }\end{array}$ & $\begin{array}{l}\text { Anak Serani gila menyabong } \\
\text { Menyabong mari di tanah rata } \\
\text { Habis di charik segenap tanjong } \\
\text { Ongas di taman indrawata }\end{array}$ & $\begin{array}{l}\text { Orang mengail lautan } \\
\text { Jelebu } \\
\text { Sarat muat ditimba ruang } \\
\text { Bagi mana mahu bertemu } \\
\text { Tuan didalam milikan } \\
\text { orang }\end{array}$ \\
\hline 4 & Banjar & $\begin{array}{l}\text { Manabung bambah di kampong } \\
\text { ganda } \\
\text { Sampa lirang kulipat-lipat } \\
\text { Lamun dandaman lawan } \\
\text { kakanda } \\
\text { Paluk guguling panggil sumangat }\end{array}$ & $\begin{array}{l}\text { Apa burung betetkah } \\
\text { Bari-bari hinggap di } \\
\text { batang } \\
\text { Apa tetapkah sudah di } \\
\text { rumah } \\
\text { Ini hari kami badatang }\end{array}$ \\
\hline 5 & Batak & $\begin{array}{l}\text { Ibuat namin pulut } \\
\text { Han hayu sidabintang } \\
\text { Hora nasiam suhut } \\
\text { Sonai homa naiundang }\end{array}$ & $\begin{array}{l}\text { Sada manukku mirah } \\
\text { Padua manuk kulabu } \\
\text { Sangapni na merinang } \\
\text { Mardilou mangan hujabu }\end{array}$ \\
\hline 6 & $\begin{array}{l}\text { Bedohai } \\
\text { Dayak } \\
\text { Taman }\end{array}$ & $\begin{array}{l}\text { Anakng pipit tecuak-cuakng } \\
\text { Cuaklah-cuakng jeRamik padi } \\
\text { Disedikit jangan dibuakng } \\
\text { Kalau dibuakng meRugi kami }\end{array}$ & $\begin{array}{l}\text { Di Riam sampai bengabut } \\
\text { Singah betamat longkokng } \\
\text { sekonau } \\
\text { Ati di dalam terasa kalut } \\
\text { Menunguk Riam menjadi } \\
\text { Rena }\end{array}$ \\
\hline 7 & Belitung & $\begin{array}{l}\text { Neq Mong dari Melake } \\
\text { Tumpa minyaq dalam } \\
\text { tempurung } \\
\text { Jangan sumbong sidaq sekae } \\
\text { Same-same anak Belitung }\end{array}$ & \\
\hline 8 & Jawa & $\begin{array}{l}\text { Koki tuwa saka Semarang } \\
\text { Tuku apem disuwir-suwir } \\
\text { Aku iya kowe kok gemang } \\
\text { Nyata gelem ayo dipikir }\end{array}$ & $\begin{array}{l}\text { Kali Srayu banyune asat } \\
\text { Urang tlaga dawa sapite } \\
\text { Kowe mau janji martobat } \\
\text { Kena apa ninggal } \\
\text { sumpahe }\end{array}$ \\
\hline 9 & $\begin{array}{l}\text { Kalimantan } \\
\text { Barat }\end{array}$ & $\begin{array}{l}\text { Biuk elang terbang tinggi } \\
\text { Inggap di kayu cendana } \\
\text { Biar remuk ancur binasa } \\
\text { Nasi sepinggan makan bedua }\end{array}$ & $\begin{array}{l}\text { Emas urai alam geleta } \\
\text { Kain pendukung carit } \\
\text { dibendi } \\
\text { Biar beurai aik mata } \\
\text { Apang inai nesik peduli } \\
\end{array}$ \\
\hline 10 & Kerinci & $\begin{array}{l}\text { Talang abang auh Cino } \\
\text { Singoah malingkah Talang } \\
\text { Kamulun } \\
\text { Badean kamal talalu hino } \\
\text { Mano diletoak badean itau }\end{array}$ & $\begin{array}{l}\text { Alam kumo rendok } \\
\text { nyemai } \\
\text { Juleang lulauk banyeak } \\
\text { ngan licain } \\
\text { Jangeang diarap pado } \\
\text { badeang kamai } \\
\text { Indauk bureak bapeak } \\
\text { musikin }\end{array}$ \\
\hline
\end{tabular}




\begin{tabular}{|c|c|c|c|}
\hline No & Region & Example 1 & Example 2 \\
\hline 11 & $\begin{array}{l}\text { Lampung } \\
\text { Pubiyan }\end{array}$ & $\begin{array}{l}\text { Way lima Padasuka } \\
\text { Kedondong kubu batu } \\
\text { Kebabangkon di sama } \\
\text { Nyak tiaram jama niku }\end{array}$ & $\begin{array}{l}\text { Relam bingi mak pedam } \\
\text { Kak liyu pukul lima } \\
\text { Badan ji lansung ngiram } \\
\text { Kesepik niku di pa }\end{array}$ \\
\hline 12 & Langkat & $\begin{array}{l}\text { Ada rakit di dalam padi } \\
\text { Mengapa tidak tuan buangkan } \\
\text { Jika sakit di dalam hati } \\
\text { Mengapa tidak tuan hilangkan }\end{array}$ & $\begin{array}{l}\text { Ada kain sungkit permata } \\
\text { Tenunan putri Galuh } \\
\text { Kencana } \\
\text { Ada lain kenangkan beta } \\
\text { Kerana beta dagang yang } \\
\text { hina }\end{array}$ \\
\hline 13 & Madura & $\begin{array}{l}\text { Kalarassa gheddhan bighi } \\
\text { Gheddihan embjuk mot- } \\
\text { penmodhan } \\
\text { Malaratta taq diq ghighi } \\
\text { Nakan sërbhuk mot-këlmodhan }\end{array}$ & $\begin{array}{l}\text { Bhako rokoq Tambhaq- } \\
\text { Aghuri } \\
\text { Sëlla cërike rokoq kretek } \\
\text { Patakoq na daq ren agun } \\
\text { Dalëm ate sëmmo netek }\end{array}$ \\
\hline 14 & Maluku & $\begin{array}{l}\text { Fia bamata - wana galima } \\
\text { Dol gia di gas fat } \\
\text { Sabakota deha bo bima } \\
\text { Pia-pia mara gabat }\end{array}$ & $\begin{array}{l}\text { Wailia wai sanana } \\
\text { Wai tamela ewa don mata } \\
\text { Biar gila liwa sinaga } \\
\text { Lal kangela tambah barasa }\end{array}$ \\
\hline 15 & $\begin{array}{l}\text { Melayu- } \\
\text { Betawi }\end{array}$ & $\begin{array}{l}\text { Rambut panjang dikasih miring } \\
\text { Ambil senapan tembak buaya } \\
\text { Sudah lama aku digiring } \\
\text { Kapan dia di tangan saya }\end{array}$ & $\begin{array}{l}\text { Kalau bukan mobil buatan } \\
\text { Tidaklah saya sampai } \\
\text { bertaksi } \\
\text { Kalau saya ingat budi tuan } \\
\text { Siang malam saya tangisi }\end{array}$ \\
\hline 16 & $\begin{array}{l}\text { Melayu/ } \\
\text { Makassar }\end{array}$ & $\begin{array}{l}\text { Kaulah semua soba sobakku } \\
\text { Pada cinta teaki ca-pa } \\
\text { Kau kudu ang-ngalle baku } \\
\text { Pada apa niaki rupa }\end{array}$ & $\begin{array}{l}\text { Bila kau jadi dowe-nu } \\
\text { Jangan hinakan tau tenaiya } \\
\text { Tindas padanya ero-ero-nu } \\
\text { Anggap mereka sangging } \\
\text { kiccia }\end{array}$ \\
\hline 17 & $\begin{array}{l}\text { Minang- } \\
\text { kabau }\end{array}$ & $\begin{array}{l}\text { Piriang putiah piriang basabun } \\
\text { Disabun anak urang Cino } \\
\text { Mamutiah bungo dalam kabun } \\
\text { Satangkai sajo nan mangilo }\end{array}$ & $\begin{array}{l}\text { Ayam hitam di anjuangan } \\
\text { Ditangkok musang malam } \\
\text { hari } \\
\text { Rindu jo dandam } \\
\text { ditangguangkan } \\
\text { Adiak bajalan tak kumbali }\end{array}$ \\
\hline 18 & Muna & $\begin{array}{l}\text { Aembulumo oropopasa } \\
\text { Akakompo nerokalei } \\
\text { Aen katoo nekakawasa } \\
\text { Miina bhala nantipalei }\end{array}$ & $\begin{array}{l}\text { Aetisamo bhela paria } \\
\text { Aforambee newesefi } \\
\text { Sapse bhela mnaria-ria } \\
\text { Psemo bhela atidolifi }\end{array}$ \\
\hline 19 & $\begin{array}{l}\text { Keturunan } \\
\text { Portugis } \\
\text { (Melaka) }\end{array}$ & $\begin{array}{l}\text { Kafrinyu kiteng santadu } \\
\text { Lanta pie bate-bate } \\
\text { Kafringyu kire anda kaju } \\
\text { Tira terban naji sake }\end{array}$ & $\begin{array}{l}\text { Pasa na bordu maar } \\
\text { Ola nabiu kere nabiga } \\
\text { Vilu vilu nangkorsan mal } \\
\text { Nungku aca dijustisa } \\
\end{array}$ \\
\hline 20 & Saluma & $\begin{array}{l}\text { Limau manis masak sepiak } \\
\text { Sesiung digunggung burung } \\
\text { Itam manis menyusul tinjak } \\
\text { Tinjak berencang puncak gunung }\end{array}$ & $\begin{array}{l}\text { Putus tali gendang serunai } \\
\text { Lubuak belimbing nga } \\
\text { buayo } \\
\text { Putus ati rindu nga punai } \\
\text { Punai di puncak kayu aro }\end{array}$ \\
\hline
\end{tabular}




\begin{tabular}{|c|c|c|c|}
\hline No & Region & Example 1 & Example 2 \\
\hline 21 & Sambas & $\begin{array}{l}\text { Dari Sambas ke Singkawang } \\
\text { Bebauru burrung di atas pohon } \\
\text { Uddah lama pemimpinnya illang } \\
\text { Ditunggu-tunggu bepuluh tahun }\end{array}$ & $\begin{array}{l}\text { Urrang Sambas pandai } \\
\text { belayar } \\
\text { Patah kemudi minggir } \\
\text { dolo kitappi } \\
\text { Uddah lama kame bisabar } \\
\text { Nunggu pindahnya Pa } \\
\text { Bupati } \\
\end{array}$ \\
\hline 22 & Serdang & $\begin{array}{l}\text { Biduk belayar di laut tenang } \\
\text { Bintang di langit nunjuk arah } \\
\text { Biar gelombang datang } \\
\text { menyerang } \\
\text { Rasa tempur tiada patah } \\
\end{array}$ & $\begin{array}{l}\text { Kembangka tikar luas-luas } \\
\text { Tempat duduk seratan } \\
\text { jerami } \\
\text { Kelih kami ini puas-puas } \\
\text { Isok pagi ho kami te sini } \\
\end{array}$ \\
\hline 23 & $\begin{array}{l}\text { Serawai } \\
\text { (Bengkulu) }\end{array}$ & $\begin{array}{l}\text { Ala kclamq mandi di ulu } \\
\text { Kami mandi di jangkaqh kagbit } \\
\text { Ala kclamaq nyadi guru } \\
\text { Kami nyadianaq murid }\end{array}$ & $\begin{array}{l}\text { Terataq batang teriti } \\
\text { Batang temeghan berang } \\
\text { sano } \\
\text { Teragaq belum bereti } \\
\text { Belum keruan bataq kato }\end{array}$ \\
\hline 24 & Sunda & $\begin{array}{l}\text { Pileuleuyan daun pulus } \\
\text { Kararas daun katumpang } \\
\text { Pileuleuyan ulah tulus } \\
\text { Masing ras ka nu di tukang }\end{array}$ & $\begin{array}{l}\text { Kasur jangkung bantal } \\
\text { panjang } \\
\text { Nya bogo dika-caikeun } \\
\text { Anu jangkung kuring- } \\
\text { melan } \\
\text { Nya bogoh urang jadikeun }\end{array}$ \\
\hline 25 & $\begin{array}{l}\text { Talang } \\
\text { Mamak }\end{array}$ & $\begin{array}{l}\text { Apit dinding berapit } \\
\text { Akan mengapit serunjunya } \\
\text { Kalau baik ambillah kapit } \\
\text { Apakan bisa sembarang gunanya }\end{array}$ & $\begin{array}{l}\text { Sedang mengkudu lagi } \\
\text { berpawal } \\
\text { Kunun pula cempedak } \\
\text { muda } \\
\text { Lagi penghulu lagi } \\
\text { bergawal } \\
\text { Kunun pula budak muda- } \\
\text { muda }\end{array}$ \\
\hline 26 & $\begin{array}{l}\text { Tanemperar } \\
\text { (Ambon) }\end{array}$ & $\begin{array}{l}\text { Yak to kufai, kal weri dalam } \\
\text { Fati la bwayar rares nde tate } \\
\text { Yak to kufai, kal urangw dalam } \\
\text { Feti la dalam nor yakw nde tate }\end{array}$ & $\begin{array}{l}\text { Yak klole far da, mpatotin } \\
\text { loran } \\
\text { Ti kwait rentes, kumdu } \\
\text { kuwate } \\
\text { Lere ma ndondam, } \\
\text { kdwaun ningw dulan } \\
\text { Dulan botuan, dulan } \\
\text { wawate }\end{array}$ \\
\hline
\end{tabular}

Chart II: Examples of regional pantun with the same structure.

However, pantun in Mandar, Toraja, Java, and the Buginese of South Sulawesi, for example, may appear with the following structure:

1. $\mathrm{a} b \mathrm{ab}$

2. $\quad \mathrm{a}$ a $\mathrm{a}$ a

3. $\mathrm{a} a \mathrm{a} b$

4. $\mathrm{a} a \mathrm{~b} b$ 
See the examples in Chart III.

\begin{tabular}{|c|c|c|c|}
\hline No & Region & Example 1 & Example 2 \\
\hline 1 & Bugis & $\begin{array}{l}\text { Lae wettue } \\
\text { Sitinajai ripakkeguna wettue } \\
\text { Au temmappakegunae wettu } \\
\text { La naritu tau kuttu } \\
\end{array}$ & $\begin{array}{l}\text { Kutue teppeuno } \\
\text { Poleana kutue temmapakbinasa } \\
\text { La kiai pasussai napasessa } \\
\text { Kuae topa mappan rasa-rasa }\end{array}$ \\
\hline 2 & Makassar & $\begin{array}{l}\text { Andik pammopporammak } \\
\text { Ia makkelongi daenta } \\
\text { Bunga ejaya } \\
\text { Niakmo mannyero kana }\end{array}$ & $\begin{array}{l}\text { Na kilo-kiloki asseng } \\
\text { Gigi bapisik bulaeng } \\
\text { Kammaki asseng } \\
\text { Tu nasuro manngukrangi }\end{array}$ \\
\hline 3 & Mandar & $\begin{array}{l}\text { Tennaq diangdi adaqna } \\
\text { Disembeang to tuna } \\
\text { Saemaq todiq } \\
\text { Di passimbeangang roppong }\end{array}$ & $\begin{array}{l}\text { La pa mala molabu } \\
\text { Labuang di kampung na } \\
\text { Bulawang randang na } \\
\text { Suasa sapparaya na }\end{array}$ \\
\hline 4 & Saluan & $\begin{array}{l}\text { Talalo kapian sina nu ahat } \\
\text { Kalu kita mongtugal bahat } \\
\text { Bali kita mohae moga'at } \\
\text { Dunia akhirat mbaha' salamat }\end{array}$ & $\begin{array}{l}\text { Kami mo'ili sonso'i hinsip } \\
\text { Sonso' nonsake mian sangga'at } \\
\text { Kalu mo'osoa boli' mohinsip } \\
\text { Kalu mosihip tantu moga'at }\end{array}$ \\
\hline 5 & Sambas & $\begin{array}{l}\text { Anak kecil di tepian pagar } \\
\text { membuat pagar di tepi } \\
\text { bangsal } \\
\text { waktu kecil malas belajar } \\
\text { sudah besar nanti menyesal }\end{array}$ & $\begin{array}{l}\text { Kalaulah lebat si buah lada } \\
\text { Pohon Tube tumbuhnya } \\
\text { condong } \\
\text { Kalaulah banyak ilmu di dada } \\
\text { jangan dicobe berlaku sombong }\end{array}$ \\
\hline 6 & Ternate & $\begin{array}{l}\text { Lahi dibu to hakka dibu } \\
\text { Dibu madaha akkee nongaai } \\
\text { Lahi nyingga to hakka } \\
\text { nyingga } \\
\text { Nyingga madaha gate gogolla }\end{array}$ & $\begin{array}{l}\text { Taggi taggi kagee na tarra } \\
\text { Hiri marri la batta batta } \\
\text { Taai taai sari to cobbo } \\
\text { Srokonnai ni giha poddo }\end{array}$ \\
\hline 7 & $\begin{array}{l}\text { Tetun } \\
\text { (Timor) }\end{array}$ & $\begin{array}{l}\text { Nanilis an ba, lakateu sina } \\
\text { Nanaak an ba, kdade malae } \\
\text { Naak atu nawai, lakaetu sina } \\
\text { Naak atu nawai, kdade malae }\end{array}$ & $\begin{array}{l}\text { Sei nee tuku ami, lidu lor nee } \\
\text { Tuku ami lidu lor, tuku mo neo } \\
\text { Ami asu oa nurak, tata hat o } \\
\text { Ami fahi oa nurak, niku hat o }\end{array}$ \\
\hline 8 & Toraja & $\begin{array}{l}\text { Allonanniko batu pirriq } \\
\text { Batu tappolo-polo } \\
\text { Poloi batu } \\
\text { Anna polo inawa }\end{array}$ & $\begin{array}{l}\text { Bunga-bunga tomambela } \\
\text { Panden torandean langiq } \\
\text { Kumpangko mai } \\
\text { Angku patikantongko }\end{array}$ \\
\hline 9 & Totoli & $\begin{array}{l}\text { I sadang ilaeng tabako } \\
\text { Mau inako-inako } \\
\text { Jagai dalan dako } \\
\text { Dalan mopido lalako }\end{array}$ & $\begin{array}{l}\text { Gaukan dei buntuna } \\
\text { Mopido kalakuanna } \\
\text { Mau namoga bukuna } \\
\text { Kalambati sarang lipuna }\end{array}$ \\
\hline
\end{tabular}

Chart III: Pantun with different structure.

This is still a preliminary survey as we have yet to document pantun from many places in Indonesia ${ }^{1}$. Thus, we have no idea whether the pantun available

1 These include: Alor, Amahal, Anggi, Apalapsili, Atambua, Ayawasi, Babo, Bade, Badung, Bajawa, Banaina, Bandanaira, Bandar, Batam Batu, Batom, Bekasi, Berau, Biak, Bintan, 
in any of the above-mentioned places are the same as the pantun we have already covered.

We also do not know whether there are "home-grown" pantun in Sri Lanka, the Cocos Islands, Suriname, Cape Town, or other places outside the Indo-Malay world with large communities of Indo-Malay peoples who have settled there through typical patterns of movement: migration, trade, marriage, employment, resettlement, deportation, and others. Politically, many Indo-Malays were expatriated to Sri Lanka and South Africa in the nineteenth century when the British and Dutch colonial masters in the Malay Peninsula and the Dutch East Indies exiled rebels from their colonies. When these peoples moved, they carried with them their language, religion, and their pantun (Muhammad Haji Salleh 2006: 9). In this way, many pantun moved from one place to another in and outside the Indo-Malay world and became an element of their identity in their newly adopted lands. Gradually these "imported" pantun are found to have been enriched with local inputs. Some of them might have undergone changes in one way or the other. In this way, the development of Minangkabau pantun outside their homelands was no different from their counterparts from elsewhere: they developed within new social and cultural surroundings, and flavoured themselves with local words or other variations only to be found in their "adopted" lands.

Allowing for minor differences in style and diction, there is occasional and isolated similarity or parallelism between some pantun in Minangkabau, Malay, and Kerinci, as shown in Chart IV.

These occurrences may be due to conscious imitations, and to the reassembling of lines, plots, and characters from various popular pantun. This is yet another interesting aspect of the pantun river and its dynamism if we were to embark on its journey and its distribution all over the IndoMalay world. As we continue to marvel at the thousands of pantun collected from different regions, we cannot help but wonder at their riches not only in terms of similarity and difference, but also with the creativity of pantun poets, whether known or unknown.

Bogor, Jayapura, Jelambar, Jember, Jimbaran, Johar Baru, Jombang, Kaimana, Kambuaya, Karangbolong, Karet, Karubaga, Kau, Kebar, Kebayoran Lama, Kediri, Keisah, Kelita, Ketapang, Kiman, Klender, Kolkata, Komodo, Kotabangun, Kuningan, Kupang, Kuta, Labuan Bajo, Labuha, Langgur, Larantuka, Lereh, Lewoleba, Longapung, Longbawan, Lunyuk, Luwuk, Madana, Madiun, Magelang, Makale, Malang, Maliana, Mampang Prapatan, Mamuju, Manado, Manggis, Mangole, Manokwari, Masamba, Mataram, Maumere, Metanguane, Menteng, Merauke, Merdey, Moanamani, Morotai Island, Moyo Island, Mulia, Muting, Nabire, Naha, Namlea, Namrole, Numfoor, Nunukan, Obano, Ocussi, Okaba, Oksibil, Palabuhanratu, Palangkaraya, Palembang, Palmerah, Palu, Pamekasan, Pancoran, Pangkalanbuun, Pangkalpinang, Parapat, Pasuruan, Payangan, Pomalaa, Poso, Purwokerto, Putussibau, Ransiki, Rantepao, Raya, Rokot, Ruteng, Samarinda, Sanana, Sanur, Sarmi, Sawu, Semarang, Senen, Serang, Serui, Singaraja, Singkep, Soroako, Sorong, Suai, Sukabumi, Sumbawa, Sunter, Surabaya, Tabanan, Taman Sari, Tambolaka, Tambora, Tanah Abang, Tanahgrogot, Tangerang, Tanjung Pandan, Tanjung Pinang, Tanjung Selor, Tarakan, Tebet, Tembagapura, Teminabuan, Timika, Tolitoli, Tuban, Ubud, Ungasan, Utara, Wagethe, Waingapu, Wamena, Waris, Wasior. 


\begin{tabular}{|l|l|l|}
\hline 1a & $\begin{array}{l}\text { Tanjung Katung airnya biru } \\
\text { Tempat orang bermandi-manda } \\
\text { Sedangkan sekampong lagikan rindu } \\
\text { Inikan pula jauh di mata }\end{array}$ & $\begin{array}{l}\text { Tanjung Katung airnya biru } \\
\text { Kalau boleh menumpang mandi } \\
\text { Hidup selalu memendam rindu } \\
\text { Bertemu denganmu mesti sekali }\end{array}$ \\
\hline 1b & $\begin{array}{l}\text { Tanjung Katung airnya biru } \\
\text { Tempat gadis berenang-renang } \\
\text { Hidup selalu menanggung rindu } \\
\text { Hanya padamu ku terkenang }\end{array}$ & $\begin{array}{l}\text { Tanjung Katung airnya biru } \\
\text { Lautnya dalam langitnya jernih } \\
\text { Hati selalu ingat padamu } \\
\text { Semakin kuat terpaut kasih }\end{array}$ \\
\hline 2 & $\begin{array}{l}\text { Memang kutahu ikan tenggiri } \\
\text { Maka kegulai dengan kelapa } \\
\text { Memang kutahu abang beristeri } \\
\text { Kumis di bibir yang menggila }\end{array}$ & $\begin{array}{l}\text { Memang kutahu ikan tenggiri } \\
\text { Selasih condong ke dalam paya } \\
\text { Memang kutahu abang beristeri } \\
\text { Kasih terdorong apakan daya }\end{array}$ \\
\hline 3 & $\begin{array}{l}\text { Bukan jantung sembarang jantung } \\
\text { Jantung pisang di dalam peti } \\
\text { Bukan bingung sembarang bingung } \\
\text { Bingung memikir si jantung hati }\end{array}$ & $\begin{array}{l}\text { Bukan jantung sembarang jantung } \\
\text { Jantung pisang di dalam peti } \\
\text { Bukan bingung sembarang bingung } \\
\text { Bingung memikirkan abang } \\
\text { mencari ganti }\end{array}$ \\
\hline 4 & $\begin{array}{l}\text { Ikan todak beranak todak } \\
\text { Ikan tenggiri mati tertimba } \\
\text { Khabar tidak berita tidak } \\
\text { Kandalah lupa kepada saya }\end{array}$ & $\begin{array}{l}\text { Ikan todak menodak-nodak } \\
\text { Menetas di dalam paya } \\
\text { Ku suruh tidak ku larang tidak } \\
\text { Kertas dan tinta abang yang punya }\end{array}$ \\
\hline
\end{tabular}

Chart IV. Parallelism between some pantun.

\section{MORE REASONS FOR THE SPREAD OF PANTUN}

As indicated, the flow of the pantun river from the past till the present has been impressive. Combining the volumes of a thousand tributaries, the mighty pantun river gathered speed and picked up force as it swirled east and south, then swungs back on itself and headed north and west. No mountain could block its growing might. Thus, there are pantun in the Hatuhaha language spoken in central Maluku in eastern Indonesia, a large and varied province. This language differs markedly from Ambon-Malay, the lingua franca of the region. Likewise, a unique aspect of Hatuhaha pantun is that their rhyme scheme is not always a b a b, but a a a as well. Nevertheless, Hatuhaha pantun are similar in contents. They too are dominated by love, compassion, advice, and fun. Called badendang kapata, pantun here are also an integral part of an oral art form involving the use of rhyming words, which, formal in manner, appears highly spontaneous in style (Sastrowardoyo and Damono 1985: 335). Another place with precipitous terrains, difficult to travel, is Alor in East Nusa Tenggara. Situated to the north of the large island of Timor, it has eight different language-groups speaking innumerable dialects. Ward (1975: 32) notes that Du Bois had collected pantun there in 1938. Its pantun, another traditional folk poetry, consists of dance-songs improvised by youths who would vie with each other in displaying their creative skills at parties that last from night to dawn. As pantun reciting is still a strong tradition, about one tenth of the adult men here are recognized singers. In the northern Moluccas 
close by, there is a small island called Ternate. It is not only well-known historically, but has also produced its share of pantun in the Ternate language. In this work, Ward (1975: 75) quotes pantun collected by Campen in 1885.

Moving elsewhere, we find that the Banjarese in South Kalimantan are noted too for being good singers as they often sing while they work. They impress one another with their elegant and harmonic pantun to express a multitude of topics, including love and religious matters. Turning our attention to northern Sumatra, we are thrilled with yet another popular oral folk poetry, called umpasa, the Simalungun-Batak term for pantun. Containing not only advices on the everyday problems of life, umpasa are also used to comment on various aspects of human life (Sastrowardoyo and Damono 1985: 53-56). Nearby at Sungaipenuh in the heart of the Kerinci country of western Jambi, there is another very rich and long pantun tradition (Ward 1975:17). Across the seas, there is yet another rich but little known pantun tradition in the Pattani region in southern Thailand. Inhabited primarily by Malays, who are either the indigenous peoples or the descendants of migrants from the neighbouring Malaysia, they are known to have established the Pattani Kingdom to replace an earlier Langkasuka Kingdom. However, Pattani is now under Thai rule through the Anglo-Siamese Treaty of 1909, whereby Thailand, then known as Siam, retained Pattani and the British controlled Kelantan, Terengganu, Kedah, and Perlis. As elsewhere, it is only natural that the peoples of the border areas intermingled and intermarried. As old and new traditions co-exist in this way, so do pantun. Thus, we have a hodgepodge of Indo-Malay pantun from different regions not only in the border areas, but also in big cities like Jakarta with its share of Betawi pantun, Kuala Lumpur, and Singapore, for instance, where different Indo-Malay ethnic groups meet and interact. Djamal Tukimin (2009) has documented Banjarese, Javanese, Minangkabau, Makasarese, and Malay pantun, for instance, that co-exist and flourish in Singapore. It is in this way that we have the luxury of interacting with a hybrid of cultural changes and adaptations in the sense that "imported" pantun change and transform others, including the "home-grown" ones. Arising from the tradition of coexisting and enriching one another, many pantun that are brought here from elsewhere have apparently been revised at some point in time to make them no longer provincial in colour, tone, and feeling. This "remaking" process might have been done by officials who first compiled collections of pantun that were transmitted orally in the nineteenth century. Whether experts can detect if a quatrain is from Aceh, Bengkulu, Kalimantan, Palembang, Makassar, Jambi, Malacca, or Singapore or elsewhere is beyond the scope of this article. Many favourite pantun went through a long odyssey of adventure up and down the Indo-Malay world with the pantun poets now and then imitating, improvising, copying and recopying as many as they would like in imitation of the American and European pop songs all over the world in our times. Their regional pantun are important to the Banjarese, Acehnese, and Minangkabau peoples in Kuala Lumpur, as one more common cultural identity after having come together after a period of separation from their 
homelands, for such purposes as the renewal of friendships, promotion of their regional values, as well as the preservation of clans and families. This implies that though no longer living in their ancestral homelands, they are linked through sharing traditional culture. Thus, get-togethers during weddings and other celebrations held from time to time are important in sustaining the cultural ties of these peoples and communities, in addition to the family ties they have through birth and marriage. This feeling of togetherness has transcended geographical boundaries. This means that it is not necessary for members of a community to be living together in their original homelands to enjoy their favourite pantun.

On the other hand, we have no explanation for the different versions of pantun in different local languages all over the Indo-Malay world. Despite their apparent difference in language, most of the pantun are similar in structure, function, and characteristics. They are different and yet the same. They are distant cousins, if not twins. The question whether they develop independently or dependently needs further research. We are not sure whether this kind of variety is the same as that in Indo-Malay architecture, food, and way of life. For example, most of the traditional houses are built on stilts using wood and other indigenous building materials, with highly angular and sloping roofs. Their variety is also due to geographic differences, while they are similar because they are related to one another in the sense that they have one thing in common: clinging to tradition. Though they vary by region, they are linked and related to one another by common characteristics.

In this paper, these different versions of pantun are likened to the tributaries of a mighty river that runs through the Indo-Malay world. That pantun river is a constant thread of identity, like DNA, that united geographically different regions down the centuries into the infinite future. But, pantun Bugis and kelong in Makassar are not the kind of pantun we are used to, see the examples in Chart V. They are different in appearance and in structure. Pantun Bugis have three lines with eight, five, and eight syllables per line while kelong Makassar have an irregular rhyme structure in all its four lines. Structurally, there are also no foreshadow (pembayang) and meaning (maksud) components that complement one another. That means that the meaning in kelong flows directly from one line to the next. It is more like tangka in Japan (information from Arena Wati, on 28 August 2007 in Makassar). Similarly, the pantun from the Sasak of Lombok are different from those in Minangkabau and Banjar, for instance. For further clarification, please refer to Chart III.

\begin{tabular}{|l|l|}
\hline Kelong Makassar & Pantun Bugis \\
\hline Merantau jauh ke negeri orang & Masemasekku muware \\
Menyeberang laut & Tatowa kuriaseng \\
Mendaki bukit & Capa' masagala \\
Kupasrah karena cinta & \\
\hline
\end{tabular}




\begin{tabular}{|l|l|}
\hline $\begin{array}{l}\text { Walaupun banyak samanya } \\
\text { Tapi samanya saja banyak } \\
\text { Namun kejutannya } \\
\text { Bagi kita tiada reda }\end{array}$ & $\begin{array}{l}\text { Anginna temmiri' tona } \\
\text { Salareng ripasenna } \\
\text { Massinala toni }\end{array}$ \\
\hline $\begin{array}{l}\text { Kutuju matainjo mange } \\
\text { Ri paranglakkeng balakna } \\
\text { Bunga rosia } \\
\text { Taraak mamo sanging bunga }\end{array}$ & $\begin{array}{l}\text { Ia teppaja kusappa } \\
\text { Rapanna rialae } \\
\text { Pallangga mariang }\end{array}$ \\
\hline
\end{tabular}

Chart V. Pantun Bugis and Kelong Makassar.

Any theory on the dissemination of pantun is incomplete without considering the highly mobile Malay, Javanese, Banjarese, and Bugis, among the many Indo-Malay peoples. Many of them have been well-known sailors, traders, and migrant labourers. When they travelled, traded, and moved from one place to another, they took their pantun along with them. It is in this way that many pantun from different places travel from one place to another. Ward (1975: 63) particularly notes the sailors of Makassar who have been famous seafarers from time immemorial. Spatially, they traded as far west as Burma, northward to the Philippines, and eastward to Polynesia, apart from the waters in the Indo-Malay world. Over time, Makassar's pantun not only grew with the community in their adopted lands and islands, but also crossbred and flourished with pantun from Java, Banjar, Minangkabau, and others. Other ways the pantun spread out was through teaching and the use of the pantun as a means of entertainment and education. People used to recite pantun not only to break the monotony of their labour, but also to lighten their burdens.

Wars and politics have also pushed the spread of the pantun. As one Malay kingdom prospered, it became the target of envy and awe of the neighbouring kingdoms. When Malacca emerged as a strong entrêpot in the fifteenth century, the pantun was already mature around the Straits of Malacca, and possibly also in Betawi, Ambon, Manado, and Makassar. As the Indo-Malay peoples grew in number, and their trade and technology improved, their domain extended. As they moved, their most favoured pantun spread with it. Similarly, with the armies that occupied new lands, their pantun spread further. After Malacca, Johor, and Aceh emerged as political and economic powers, and as their trade and commerce expanded, their pantun were carried to even more distant lands and islands. However, the process of the dissemination of pantun was made even more intense with the development of trade and commerce. On that note, Muhammad Haji Salleh (2006: 8-9) reasoned that the very complex networks of maritime voyages and trading undertaken by the Buginese, Malay, Javanese, Minangkabau, and Bajau, for instance, helped to transport the pantun throughout the Indo-Malay world. Thus we find Buginese, Malay, Javanese, and Minangkabau pantun in different parts of the Indo-Malay world. In the past, pantun were firmly based in agricultural society, supplemented by sophisticated maritime trade and commerce working closely together to form a solid economic basis for prosperity in the Indo-Malay world. These 
developments mark important chapters in the history of the dissemination of pantun over the Indo-Malay world. In this development, we find that when people from different ethnic groups came together, they lived harmoniously with their neighbours at different stretches of the pantun river. Following increasingly common inter-ethnic marriages, pantun from different ethnic groups enriched one another. In this way, regional pantun expanded.

\section{CONCLUSION}

Pantun are extremely popular among the Indo-Malay peoples. Unlike any other form of poetry, it is not restricted in its use to a particular class or social group, and thus has become the most common way of giving advice, showing respect, and expressing pleasure. In the past when there were not too many sources of information, education, or entertainment, the rich pantun fulfilled the people 's needs for these matters. As a result, the pantun has become an integral part of almost every Indo-Malay social and cultural activity. Advice, education, values, and philosophy as expressed in the pantun are no less important than those in proverbs and customary sayings. In terms of function, collectively they offer general insights into human problems, including conflicts and dilemmas and more importantly suggest how best to solve them and thus contribute to our understanding of Indo-Malay man, life, nature, justice, and knowledge. Looking back at them, the extant pantun really form a wonderful collective heritage, wisdom, and memory of the Indo-Malay civilizations. Though the birthplace of the pantun river is still a mystery to us at the other end of the time tunnel, we can plot the vast sweep of the river as it cuts across the entire breadth and width of the Indo-Malay world, after documenting different pantun from different regions in different dialects. From the vintage point of documentation, that mighty pantun river and its tributaries writhe and curl back on themselves, bend in wild changes of course as they spill wildly from earliest known Indo-Malay settlements to big metropolis like Jakarta and Kuala Lumpur in the 21st century. Its great bend sweeps north to Manado, down through Mandar to the rich flatlands of Lampung. Its course, traced roughly following the principal rivers, cuts across ethnic, religious, linguistic, and other social-economic barriers.

Though things are changing fast with technology, the pantun still receive great loving care and admiration. Having survived the collapse of the IndoMalay kingdoms in the nineteenth century at the hands of Dutch and British colonial masters, the pantun river holds promises for our future, as there is still much to be learned of our past that is locked in the vaults of pantun tradition and culture. Among them are advices, ideals, philosophies, duties, loyalties, ethics, family, respect, filial duties, obedience, memories, and many others that have gathered collectively in the pantun over the centuries. The volume and the variety of their contents as they traversed down the centuries give the pantun its golden glow that will shine for a long time to come. The spread of pantun in different names in different languages in the Indo-Malay world also illustrates vividly why the pantun river bears its attraction on the local 
people from the past to the present day. By the time it reached us in the 21st century, it carries an immense load of wisdom, culture, and literary value. As such, the glory of the pantun has never become lost in time.

\section{REFERENCES}

Daillie, François-René. 1988. Alam Pantun Melayu; Studies on the Malay Pantun. Kuala Lumpur: Dewan Bahasa dan Pustaka.

Ding Choo Ming. 2008a. Pantun Peranakan Baba:Mutiara Gemilang Negeri-Negeri Selat. Bangi: Penerbit Universiti Kebangsaan Malaysia.

Ding Choo Ming. 2008b. "The role of pantun as cultural identity for Nusantara in the 21st century and beyond". Paper, The seventh Workshop of the API Fellowships, University of Gadjah Mada, Yogyakarta, 23-26 November.

Ding Choo Ming. 2008c. "Parikan/Wangsalan-Pantun Java", Portal ATMA; Senada Bol. 315 (April): 16-17.

Muhammad Haji Salleh. 2006. Romance and laughter in the Archipelago; Essays on classical and contemporary poetics of the Malay world. Pulau Pinang: Penerbit Universiti Sains Malaysia (USM).

Muhammad Haji Salleh and Bazrul Bahaman. 1999. "Sejarah kritis asal-usul perkembangan, sebaran bentuk, estetika pantun Nusantara". Laporan Kajian IRPA, ATMA, UKM.

Tukimin, Djamal. 2009. "Pendokumentasian pantun di Singapura". Laporan penyelidikan sebagai karyawan tamu di Institut Alam dan Tamadun Melayu, UKM, Bangi.

Tweddell, Collin E. and Linda Amy Kimball. 1985. Introduction to the peoples and cultures of Asia. New York: Prentice-Hall.

Winstedt, R.O. 1969. A history of classical Malay literature. Kuala Lumpur/ Oxford: Oxford University Press.

Sastrowardoyo, Subagio and Sapardi Djoko Damono (eds). 1985. Anthology of ASEAN literatures. Jakarta: ASEAN Committee on Culture and Information.

Ward, Philip. 1975. Indonesian traditional poetry. New York: Oleander Press. 